\title{
BMJ Open Risk factors and between-hospital variation of caesarean section in Denmark: a cohort study
}

\author{
Sonja Wehberg, ${ }^{1,2}$ Rikke Guldberg, ${ }^{1,2}$ Kim Oren Gradel, ${ }^{1,2}$ Ulrik Schiøler Kesmodel, ${ }^{3}$ \\ Lis Munk, ${ }^{4}$ Charlotte Brix Andersson, ${ }^{5}$ Line Riis Jølving, ${ }^{1,2}$ Jan Nielsen, ${ }^{1,2}$ \\ Bente Mertz Nørgård ${ }^{1,2}$
}

To cite: Wehberg $S$, Guldberg R, Gradel K0, et al. Risk factors and between-hospital variation of caesarean section in Denmark: a cohort study. BMJ Open 2018;8:e019120. doi:10.1136/ bmjopen-2017-019120

- Prepublication history and additional material for this paper are available online. To view these files, please visit the journal online (http://dx.doi. org/10.1136/bmjopen-2017019120).

Received 14 August 2017 Revised 30 0ctober 2017 Accepted 15 December 2017

Check for updates

${ }^{1}$ Center for Clinical

Epidemiology, Odense University Hospital, Odense, Denmark

${ }^{2}$ Research Unit of Clinical

Epidemiology, Institute of

Clinical Research, University

of Southern Denmark, Odense,

Denmark

${ }^{3}$ Department of Obstetrics and Gynecology, Herlev University Hospital, Herlev, Denmark

${ }^{4}$ Swedish Association for Health Professionals, Stockholm,

Sweden

${ }^{5}$ Department of Obstetrics and Gynecology, Aalborg University Hospital, Aalborg, Denmark

Correspondence to

Dr Sonja Wehberg;

sonja.wehberg@rsyd.dk

\section{ABSTRACT}

Objectives The aim of this study was to estimate the effects of risk factors on elective and emergency caesarean section (CS) and to estimate the betweenhospital variation of risk-adjusted CS proportions. Design Historical registry-based cohort study. Settings and participants The study was based on all singleton deliveries in hospital units in Denmark from January 2009 to December 2012. A total of 226612 births by 198590 mothers in 29 maternity units were included. Primary and secondary outcome measures We estimated (1) OR of elective and emergency CS adjusted for several risk factors, for example, body mass index, parity, age and size of maternity unit and (2) risk-adjusted proportions of elective and emergency CS to evaluate between-hospital variation.

Results The CS proportion was stable at $20 \%-21 \%$, but showed wide variation between units, even in adjusted models. Large units performed significantly more elective CSs than smaller units, and the risk of emergency CS was significantly reduced compared with smaller units. Many of the included risk factors were found to influence the risk of CS. The most important risk factors were breech presentation and previous CS. Four units performed more CSs and one unit fewer CSs than expected.

Conclusion The main risk factors for elective CS were breech presentation and previous CS; for emergency CS they were breech presentation and cephalopelvic disproportion. The proportions of CS were stable during the study period. We found variation in risk-adjusted CS between hospitals in Denmark. Although exhaustive models were applied, the results indicated the presence of systematic variation between hospital units, which was unexpected in a small, well-regulated country such as Denmark.

\section{INTRODUCTION}

Caesarean section (CS) is one of the essential factors in reducing the risk of intrapartum fetal death. Nevertheless, CS can also cause several complications. For the mother, surgical complications such as bleeding, infection and thrombosis may occur, ${ }^{1}$ and the risk of uterine rupture and placenta praevia in subsequent pregnancies is increased. ${ }^{2}$
Strengths and limitations of this study:

- Our study was population-based, covering four calendar years and a high number of deliveries and investigating the effects of a wide range of risk factors for elective caesarean section (CS) and emergency CS, both maternal-related, fetal-related, labour-related and unit-related factors.

- The Danish Medical Birth Register includes data on all births at hospital maternity units, where $99 \%$ of all Danish births take place.

- This study has all the limitations inherent to a historical registry-based cohort study design, where not all potential maternal or neonatal risk factors were available and where the coding of events was done after the end of delivery.

- The lack of knowledge about the actual decision for CS is another weakness of this study. Specifically, the term 'emergency CS' covers a broad range of situations in a maternity ward, as emergency CS is seen as a homogenous group and distinctions are not made between degrees of urgency.

- Data for this study comes from one country in Northern Europe only, and Denmark is a small, well-regulated country with equal and free access to health services. Even though data homogeneity might be regarded as strength, transferability of the results to other countries is limited.

Overall, CS has been associated with a higher risk of severe maternal morbidity and mortality when compared with vaginal delivery. ${ }^{3}$ For the child, in general there is a higher risk of neonatal complications such as respiratory distress syndrome, pulmonary hypertension and iatrogenic prematurity as these conditions occur more often after CS, ${ }^{14}$ although CS in some selected situations, such as prematurity, can reduce neonatal mortality and morbidity. ${ }^{5}$

The average proportion of CS has increased dramatically in many countries over the last three decades. ${ }^{6-8}$ The WHO states that at population level, CS rates higher than 
$10 \%$ are not associated with reductions in maternal and newborn mortality rates, ${ }^{9}$ and other studies suggest that proportions above this limit may do more harm than good. ${ }^{10}{ }^{11}$ In Denmark, the CS proportion reached $21 \%$ in $2012^{12}$ when compared with $5 \%$ in $1973 .{ }^{13}$ Although this rise has been influenced by many factors such as the increasing age of nulliparous women, the increasing number of pregnant women with previous CS, a greater maternal preference for CS, changes in women's clinical risk profiles (eg, higher prevalence of pre-existing diseases/obesity), ${ }^{14}$ and improved neonatal outcomes, the reasons for the steep rise remain unexplained.

Besides this general increase in CS, a large variation between countries, ${ }^{11} 1516$ regions $^{7}$ and hospitals ${ }^{6}{ }^{17}$ has been documented. The variation for emergency CS has been reported to be larger than that for elective CS. ${ }^{6}$ Even when proportions were risk-adjusted ${ }^{6}$ or restricted to subgroups of women, ${ }^{18} 19$ the large variation tended to persist, which suggests a strong systematic component. Systematic variation between units could have occurred if key factors were missed in the risk adjustment and/or guidelines on CS decision were not sufficiently defined or followed to the letter. ${ }^{7}$ In Denmark, there is no national guideline covering decision making for $\mathrm{CS}$ in general. In Denmark, the CS proportions for 2012 varied between hospital units, ranging from $5 \%$ to $27 \%,{ }^{12}$ but the results for risk-adjusted proportions have not been published so far. On the basis of the population-based Danish registry data, we set out to define exhaustive models for the probability of elective or emergency CS, covering a wide range of potential risk factors.

This study aimed to (1) estimate associations between risk factors and CS rates, and (2) assess the between-hospital variation of the risk-adjusted CS proportions.

\section{METHODS}

\section{Data sources}

The data source was the Danish Medical Birth Register, which was established in 1973 and includes data on all births at hospital maternity units, where $99 \%$ of all Danish births take place. The registry contains information on, for example, parity, birth weight, gestational age, diagnoses regarding pre-pregnancy risk factors, medical diseases, and complications and interventions during pregnancy and delivery. The recorded information is based largely on diagnostic codes such as those found in the International Classification of Diseases, 10th Revision (ICD-10) and procedure codes such as those in the Nordic Medico-Statistical Committee classification of surgical procedures. Data from the register are available for researchers on request to the Danish Health Data Authority, and specified data required for the study are delivered pseudo anonymised.

The study period was from 1 January 2009 to 31 December 2012, during which time there were 229041 singleton births in 32 different maternity units. This number includes both live and stillborn children from gestational week 24 and excludes births after abortion procedure. Births were subsequently excluded if they were recorded (1) in very small units with fewer than 100 births per year (in total 28 births from three units), (2) with invalid code for the maternity unit ( $\mathrm{n}=33$ births), (3) with missing or incorrect birth diagnosis $(n=251)$, (4) with inconclusive mode of delivery $(n=1766)$ and (5) for mothers younger than 15 or older than 44 years of age, limits inspired by similar limits in a prior study ${ }^{10}(\mathrm{n}=351)$. Thus, 226612 singleton births (98.9\%) by 198590 mothers in 29 maternity units were included in the study; 27651 women had more than one singleton birth during the study period.

\section{Outcome measures}

The mode of delivery was classified as elective CS, emergency CS or vaginal birth (online supplementary table S1), where all non-elective CSs were classified as emergency CS. First, we analysed the proportion of-and risk for-elective CS among all singleton deliveries, and second, the proportion of-and risk for-emergency CS among all singleton deliveries after excluding those by elective CS. Finally, similar analyses were performed in a low-risk subgroup of nulliparous women giving birth to a child in cephalic presentation at $\geq 37$ weeks +0 days in spontaneous labour (definition comparable to Robson's group $\left.1^{20}\right)$.

\section{Risk factors}

A number of risk factors for CS were included. These were maternal-related factors: age, prenatal smoking, body mass index (BMI), maternal height below $163 \mathrm{~cm}$, parity and the complication factors of diabetes (both gestational and pre-existing), hypertension, pre-eclampsia/eclampsia, placenta praevia (which might have been recorded in gestational week 20 at an ultrasound examination and thus may not represent praevia at the time of labour)/placental abruption and premature rupture of membranes; fetal-related factors: gestational age (in weeks+days), sex and macrosomia (birth weight above $4500 \mathrm{~g}$ ); labour-related factors: augmentation of labour with oxytocin infusion during delivery, induction of labour, fetal presentation, cephalopelvic disproportion and fetal distress; and maternity unit-related factors: size of maternity unit based on numbers of births per year, weekend delivery (Saturday, Sunday and all national holidays) and night delivery (deliveries between 20:00 to 7:59). In Denmark, an elective CS is scheduled on weekdays in the day time (usually between 8.00 and 15:30). Missing values of risk factors were treated as no exposure. For detailed definitions of the risk factors and their categorisation see online supplementary table S2.

\section{Statistical analysis}

To estimate the effect of the risk factors on the probability of elective or emergency CS, three separate multivariable logistic regression models were fitted, with clusters taken into account. They were used to define risk-adjusted 
Table 1 Elective CS: distribution of singleton deliveries and proportion of elective CS according to risk factors, all included in a multivariable logistic regression model

\begin{tabular}{|c|c|c|c|c|}
\hline \multirow[b]{3}{*}{ Singleton deliveries } & \multirow{2}{*}{$\begin{array}{l}\text { All deliveries } \\
\mathrm{n}(\%) \\
\end{array}$} & \multicolumn{3}{|l|}{ Elective CS } \\
\hline & & n (\% within category) & OR & $95 \% \mathrm{Cl}$ \\
\hline & $226612(100)$ & $19620(8.7)$ & & \\
\hline \multicolumn{5}{|l|}{ Year } \\
\hline 2009 & $58880(27)$ & $5186(8.8)$ & Ref & \\
\hline 2010 & $59139(27)$ & $4973(8.4)$ & 0.94 & 0.89 to 0.99 \\
\hline 2011 & $55099(24)$ & $4773(8.7)$ & 0.92 & 0.87 to 0.97 \\
\hline 2012 & $53574(24)$ & $4688(8.8)$ & 0.95 & 0.90 to 1.00 \\
\hline \multicolumn{5}{|l|}{ Size of maternity unit } \\
\hline Small & $13638(6)$ & $884(6.5)$ & 0.74 & 0.68 to 0.81 \\
\hline Medium & $97653(43)$ & $8746(9.0)$ & Ref & \\
\hline Large & $115321(51)$ & $9990(8.7)$ & 1.08 & 1.04 to 1.12 \\
\hline Weekend delivery (yes) & $60993(27)$ & $260(0.4)$ & 0.03 & 0.03 to 0.04 \\
\hline Night delivery (yes) & $100718(44)$ & $361(0.4)$ & 0.02 & 0.02 to 0.02 \\
\hline \multicolumn{5}{|l|}{ Maternal age (years) } \\
\hline $15-19$ & $3292(1)$ & $93(2.8)$ & 0.62 & 0.50 to 0.77 \\
\hline 20-34 & $176722(78)$ & $13179(7.5)$ & Ref & \\
\hline $35-44$ & $46598(21)$ & $6348(13.6)$ & 1.58 & 1.51 to 1.66 \\
\hline Maternal smoking (yes) & $28150(12)$ & $2157(7.7)$ & 0.99 & 0.93 to 1.05 \\
\hline \multicolumn{5}{|l|}{ Maternal BMI } \\
\hline $14.0-18.4$ & $9321(4)$ & $642(6.9)$ & 1.02 & 0.92 to 1.13 \\
\hline $18.5-24.9$ & $142597(63)$ & $10986(7.7)$ & Ref & \\
\hline $25.0-29.9$ & 46731 (21) & $4593(9.8)$ & 1.17 & 1.11 to 1.22 \\
\hline $30-34.9$ & $18291(8)$ & 2093 (11.4) & 1.37 & 1.29 to 1.47 \\
\hline $35.0+$ & $9672(4)$ & $1306(13.5)$ & 1.56 & 1.43 to 1.70 \\
\hline Maternal height $\leq 163 \mathrm{~cm}$ (yes) & $55288(24)$ & $5389(9.7)$ & 1.05 & 1.00 to 1.09 \\
\hline \multicolumn{5}{|l|}{ Parity } \\
\hline Nulliparous & $104448(46)$ & $5299(5.1)$ & 1.08 & 1.03 to 1.14 \\
\hline Multiparous without previous CS & $96166(42)$ & $3855(4.0)$ & Ref & \\
\hline Multiparous with previous CS & $25998(11)$ & 10466 (40.3) & 16.9 & 16.08 to 17.75 \\
\hline \multicolumn{5}{|l|}{ Complication factors } \\
\hline Diabetes & $422(0.2)$ & $42(10.0)$ & & \\
\hline Hypertension & $3954(2)$ & $356(9.0)$ & & \\
\hline Pre-eclampsia/eclampsia & $6257(3)$ & $385(6.2)$ & & \\
\hline Placenta praevia & $1128(0.5)$ & $511(45.3)$ & & \\
\hline Placental abruption & $938(0.4)$ & $16(2)$ & & \\
\hline Premature rupture of membranes & $17548(8)$ & $98(0.6)$ & & \\
\hline Complication (yes) & $29461(13)$ & $1374(4.7)$ & 0.46 & 0.42 to 0.50 \\
\hline \multicolumn{5}{|l|}{ Term } \\
\hline$<37+0$ & $10992(5)$ & $615(5.6)$ & 0.28 & 0.25 to 0.32 \\
\hline $37+0-41+6$ & 205338 (91) & $18897(9.2)$ & Ref & \\
\hline$\geq 42+0$ & $10282(5)$ & $108(1.1)$ & 0.12 & 0.10 to 0.15 \\
\hline Female baby (yes) & $110397(49)$ & $9812(8.9)$ & 1.01 & 0.97 to 1.05 \\
\hline Breech presentation (yes) & $8979(4)$ & $4382(48.8)$ & 24.02 & 22.45 to 25.70 \\
\hline Macrosomia (yes), >4500 g & $6220(3)$ & $578(9.3)$ & 1.11 & 0.98 to 1.25 \\
\hline
\end{tabular}

OR with $95 \% \mathrm{Cl}$ based on a multivariable logistic regression model.

BMI, body mass index; CS, caesarean section; n, number; Ref, reference group. 
Table 2 Emergency CS: distribution of deliveries (all singleton deliveries without deliveries by elective CS) and proportion of emergency CS according to risk factors

\begin{tabular}{|c|c|c|c|c|}
\hline \multirow[b]{3}{*}{ Singleton deliveries minus elective CS } & \multirow{2}{*}{$\begin{array}{l}\text { Deliveries } \\
\mathrm{n}(\%) \\
\end{array}$} & \multicolumn{3}{|l|}{ Emergency CS } \\
\hline & & n (\% within category) & OR & $95 \% \mathrm{Cl}$ \\
\hline & $206992(100)$ & 26305 (12.7) & & \\
\hline \multicolumn{5}{|l|}{ Year } \\
\hline 2009 & $53614(27)$ & $7003(13.1)$ & Ref & \\
\hline 2010 & $54166(27)$ & $7023(13.0)$ & 0.96 & 0.92 to 1.00 \\
\hline 2011 & $50326(24)$ & $6271(12.5)$ & 0.90 & 0.86 to 0.94 \\
\hline 2012 & $48886(24)$ & $6008(12.3)$ & 0.93 & 0.89 to 0.98 \\
\hline \multicolumn{5}{|l|}{ Size of maternity unit } \\
\hline Small & $12754(6)$ & $1464(11.5)$ & 1.02 & 0.95 to 1.09 \\
\hline Medium & 88907 (43) & $11292(12.7)$ & Ref & \\
\hline Large & $105331(51)$ & $13549(12.9)$ & 0.90 & 0.87 to 0.93 \\
\hline Weekend delivery (yes) & $60733(29)$ & 7763 (12.8) & 1.00 & 0.97 to 1.03 \\
\hline Night delivery (yes) & $100357(48)$ & 12046 (12.0) & 0.93 & 0.90 to 0.95 \\
\hline \multicolumn{5}{|l|}{ Maternal age (years) } \\
\hline $15-19$ & $3199(2)$ & $309(9.7)$ & 0.60 & 0.52 to 0.69 \\
\hline $20-34$ & $163543(79)$ & 20034 (12.3) & Ref & \\
\hline $35-44$ & $40250(19)$ & $5962(14.8)$ & 1.48 & 1.42 to 1.53 \\
\hline Maternal smoking (yes) & 25993 (13) & $3571(13.7)$ & 1.10 & 1.05 to 1.15 \\
\hline \multicolumn{5}{|l|}{ Maternal BMI } \\
\hline $14.0-18.4$ & $8679(4)$ & $774(8.9)$ & 0.77 & 0.70 to 0.84 \\
\hline $18.5-24.9$ & $131611(64)$ & $14658(11.1)$ & Ref & \\
\hline $25.0-29.9$ & $42138(20)$ & $6147(14.6)$ & 1.34 & 1.29 to 1.39 \\
\hline $30-34.9$ & $16198(8)$ & $2929(18.1)$ & 1.75 & 1.66 to 1.84 \\
\hline $35.0+$ & $8366(4)$ & $1797(21.5)$ & 2.07 & 1.94 to 2.22 \\
\hline Maternal height $\leq 163 \mathrm{~cm}$ (yes) & $49899(24)$ & $8701(17.4)$ & 1.67 & 1.62 to 1.73 \\
\hline \multicolumn{5}{|l|}{ Parity } \\
\hline Nulliparous & $99149(48)$ & $16712(16.9)$ & Ref & \\
\hline Multiparous without previous CS & $92311(45)$ & $3985(4.3)$ & 0.25 & 0.24 to 0.27 \\
\hline Multiparous with previous CS & $15532(8)$ & $5608(36.1)$ & 2.97 & 2.84 to 3.10 \\
\hline \multicolumn{5}{|l|}{ Complication factors } \\
\hline Diabetes & $380(0.2)$ & $74(19.5)$ & & \\
\hline Hypertension & $3598(2)$ & 707 (19.6) & & \\
\hline Pre-eclampsia/eclampsia & $5872(3)$ & $2110(35.9)$ & & \\
\hline Placenta praevia & $617(0.3)$ & $420(68.1)$ & & \\
\hline Placental abruption & $967(0.5)$ & $622(64.3)$ & & \\
\hline Premature rupture of membranes & $17450(8)$ & $3536(20.3)$ & & \\
\hline Complication (yes) & $28087(14)$ & $7129(25.4)$ & 1.98 & 1.90 to 2.06 \\
\hline Augmentation of labour (yes) & $59228(29)$ & $9825(16.6)$ & 1.01 & 0.98 to 1.05 \\
\hline Any induced labour (yes) & 38767 (19) & $7128(18.4)$ & 1.40 & 1.35 to 1.46 \\
\hline Induced by medication & $27166(13)$ & $5303(19.5)$ & & \\
\hline Induced by operation & $21359(10)$ & $3882(18.2)$ & & \\
\hline \multicolumn{5}{|l|}{ Term } \\
\hline$<37+0$ & $10377(5)$ & $3823(36.8)$ & 2.94 & 2.77 to 3.12 \\
\hline $37+0-41+6$ & $186441(90)$ & $20400(10.9)$ & Ref & \\
\hline
\end{tabular}




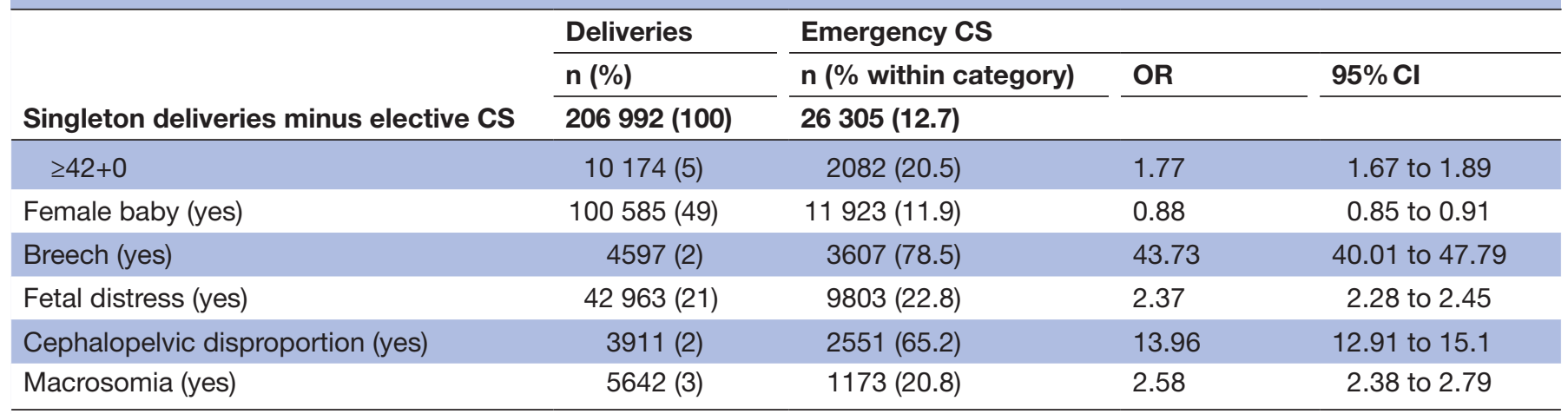

OR with $95 \% \mathrm{Cl}$ based on a multivariable logistic regression model.

BMI, body mass index; CS, caesarean section; n, Number; Ref, reference group.

proportions as follows. Summing up the probabilities predicted by the logistic model over deliveries for each maternity unit yielded the predicted proportion per unit. Dividing the observed proportion per unit by the predicted proportion multiplied by the mean national proportion yielded the risk-adjusted proportion per maternity unit. Funnel plots ${ }^{21}$ were created for risk-adjusted proportions for both elective and emergency CS; the basis for comparison and confidence was the mean national unadjusted proportion. To incorporate heterogeneity, funnel plot limits were adjusted by an additive overdispersion term (ie, an estimate of the between-unit variability, reported as SD in \%) based on a random-effects model approach. ${ }^{22}$ The corresponding test of heterogeneity between maternity units was reported. ${ }^{22}$ In addition, variation coefficients between maternity units were calculated. A coefficient of variation is defined as the SD divided by the mean. A $P$ value below 0.05 was considered statistically significant. All analyses were performed using Stata software, version 13 (Stata LP).

\section{RESULTS}

Analyses were based on 226612 singleton births. The number of births per maternity unit ranged from 383 to 11300 . There was a $9 \%$ decrease of singleton births in Denmark during the study period, from a total of 58880 in 2009 to 53574 in 2012 (table 1). In total, about a fifth $(\mathrm{n}=45925,20.3 \%)$ ended in CS, including 19620 (8.7\%) elective CSs (tables 1 and 2). Both the overall CS proportion and the proportion of elective CS remained stable at about $20 \%$ and $9 \%$, respectively (tables 1 and 2).

The risk of emergency CS remained stable across the study period for small and medium-sized units, while the risk decreased significantly from 2009 to 2010 for large maternity units and thereafter remained stable (results not shown).

\section{Risk factors for CS}

The multivariable ORs of all risk factors for elective CS included in the logistic model are presented in table 1. All the included risk factors had an impact on the risk of elective CS except for maternal smoking, maternal height, macrosomia and sex. The most important risk factors were breech presentation (OR 24.02 (95\% CI 22.45 to 25.7)) and previous CS (OR 16.9 (95\% CI 16.08 to 17.75$)$ ). Elective CS was the mode of delivery for $50 \%$ of all breech presentations and for $40 \%$ with previous CS.

The multivariable ORs of all risk factors for emergency CS are presented in table 2. Overall, the mean proportion of emergency CS was $12.7 \%$. Over the 4-year study period, the proportion of emergency CS fell slightly but consistently from $13.1 \%$ in 2009 to $12.3 \%$ in 2012 (OR 0.93 (95\% CI 0.89 to 0.98 ), using 2009 as reference). The majority of the included risk factors influenced the risk of emergency CS. The most important risk factors were breech presentation (OR 43.73 (95\% CI 40.01 to 47.79)) and cephalopelvic disproportion (OR 13.96 (95\% CI 12.91 to 15.10$)$ ). The risk for emergency CS remained unchanged regardless of the day of the week (OR 1.00 (95\% CI 0.97 to 1.03)), but was slightly less at night compared with daytime (OR 0.93 (95\% CI 0.90 to 0.95 )).

Breech presentations with intended vaginal delivery ended with emergency CS in almost $80 \%$ of the cases. About two-thirds of the women with intended vaginal delivery after previous CS succeeded in having vaginal birth. Nonetheless, the corresponding risk for emergency CS was significantly higher for these women compared with that for nulliparous women (OR 2.97 (95\% CI 2.84 to $3.10)$ ). The risk for emergency CS was lowest for multiparous women without previous CS (OR 0.25 (95\% CI 0.24 to 0.27$)$ ).

The (recorded) occurrence of maternal complication factors ranged from below $1 \%$ for diabetes and placenta praevia/placental abruption to $2 \%-3 \%$ for hypertension and pre-eclampsia/eclampsia up to $8 \%$ for premature rupture of membranes. In these groups, the proportion of emergency CS was at least about $20 \%$ for diabetes, hypertension and membrane rupture; $36 \%$ for pre/eclampsia; $64 \%$ for placenta praevia; and $68 \%$ for placental abruption. The OR for the combined complication factor was 1.98 (95\% CI 1.90 to 2.06$)$. In this study population, there were 1128 women with reported placenta praevia, and of 
Table 3 Emergency CSs in a low-risk subgroup (nulliparous women who gave birth to a child in cephalic presentation at $\geq 37$ weeks +0 days in spontaneous labour)

\begin{tabular}{|c|c|c|c|c|}
\hline \multirow[b]{3}{*}{ Singleton deliveries, subgroup } & \multirow{2}{*}{$\begin{array}{l}\text { Subgroup, all } \\
\mathbf{n} \text { (\% of all) }\end{array}$} & \multicolumn{3}{|l|}{ Emergency CS } \\
\hline & & n (\% within category) & OR & $95 \% \mathrm{Cl}$ \\
\hline & $74002(100)$ & \multicolumn{3}{|l|}{$8698(11.8)$} \\
\hline \multicolumn{5}{|l|}{ Year } \\
\hline 2009 & $19353(26)$ & 2357 (12.2) & Ref & \\
\hline 2010 & $19186(26)$ & $2338(12.2)$ & 0.95 & 0.89 to 1.02 \\
\hline 2011 & $17884(24)$ & $1994(11.2)$ & 0.87 & 0.81 to 0.93 \\
\hline 2012 & $17579(24)$ & 2009 (11.4) & 0.94 & 0.88 to 1.01 \\
\hline \multicolumn{5}{|l|}{ Size of maternity unit } \\
\hline Small & $4343(6)$ & $543(12.5)$ & 1.02 & 0.92 to 1.13 \\
\hline Medium & $27970(38)$ & 3416 (12.2) & Ref & \\
\hline Large & $41689(56)$ & 4739 (11.4) & 0.82 & 0.77 to 0.86 \\
\hline Weekend delivery (yes) & $22516(30)$ & $2672(11.9)$ & 1.02 & 0.97 to 1.08 \\
\hline Night delivery 20:00-07:59 (yes) & $35380(48)$ & 4239 (12.0) & 1.05 & 1.00 to 1.10 \\
\hline \multicolumn{5}{|l|}{ Maternal age (years) } \\
\hline $15-19$ & $2282(3)$ & $158(6.9)$ & 0.65 & 0.55 to 0.78 \\
\hline 20-34 & $64080(87)$ & $7106(11.1)$ & Ref & \\
\hline $35-44$ & $7640(10)$ & $1434(18.8)$ & 1.76 & 1.64 to 1.89 \\
\hline Maternal smoking (yes) & $9708(13)$ & $1143(11.8)$ & 1.04 & 0.97 to 1.12 \\
\hline \multicolumn{5}{|l|}{ Maternal BMI } \\
\hline $14.0-18.4$ & $3644(5)$ & $275(7.6)$ & 0.75 & 0.66 to 0.86 \\
\hline $18.5-24.9$ & $50370(68)$ & 5245 (10.4) & Ref & \\
\hline 25.0-29.9 & $13432(18)$ & 1959 (14.6) & 1.40 & 1.32 to 1.49 \\
\hline $30-34.9$ & $4572(6)$ & 789 (17.3) & 1.76 & 1.61 to 1.92 \\
\hline $35.0+$ & $1984(3)$ & $430(21.7)$ & 2.18 & 1.93 to 2.46 \\
\hline Maternal height $\leq 163 \mathrm{~cm}$ (yes) & $17698(24)$ & 3008 (17.0) & 1.78 & 1.69 to 1.88 \\
\hline Complication (yes) & $7880(11)$ & $1572(19.9)$ & 1.98 & 1.85 to 2.11 \\
\hline Augmentation of labour (yes) & $32219(44)$ & $5411(16.8)$ & 1.57 & 1.49 to 1.66 \\
\hline \multicolumn{5}{|l|}{ Term } \\
\hline $37+0-41+6$ & $71322(96)$ & 8089 (11.3) & Ref & \\
\hline$\geq 42+0$ & $2680(4)$ & $609(22.7)$ & 1.96 & 1.77 to 2.18 \\
\hline Female baby (yes) & $36265(49)$ & $3877(10.7)$ & 0.87 & 0.83 to 0.91 \\
\hline Fetal distress (yes) & $22086(30)$ & $4311(19.5)$ & 2.38 & 2.26 to 2.51 \\
\hline Cephalopelvic disproportion (yes) & $2093(3)$ & $1293(61.8)$ & 13.12 & 11.89 to 14.48 \\
\hline Macrosomia (yes) & $1139(2)$ & $381(33.4)$ & 3.36 & 2.92 to 3.87 \\
\hline
\end{tabular}

Distribution of subgroup births and proportion of emergency CS according to risk factors. OR with $95 \% \mathrm{Cl}$ based on a multivariable logistic regression model.

BMI, body mass index; CS, caesarean section; n, number; Ref, reference group.

these, 932 (83\%) underwent CS. An elective procedure was recorded for $511(45 \%)$ of these women.

The results for emergency CS in the low-risk nulliparous subgroup are presented in table 3 . The risk factors were largely distributed as in the full dataset. However, women in the subgroup were younger on average (mean age 28.3 compared with 30.1 in the full dataset), relatively more women had augmentation of labour ( $44 \%$ vs $29 \%)$, and fetal distress was reported more often (30\% vs $21 \%)$.
The mean proportion of emergency CS was about $1 \%$ lower in the low-risk subgroup $(11.8 \%)$ than in the full dataset. Overall, the emergency CS proportions with respect to risk factors in the subgroup were within $\pm 5 \%$ of those in the full dataset, except for macrosomia $(33.4 \%$ emergency CS in the subgroup as compared with $20.8 \%$ in the full dataset). Moreover, the ORs resulting from the logistic model in the subgroup were predominantly similar to those of the full dataset. However, the ORs 

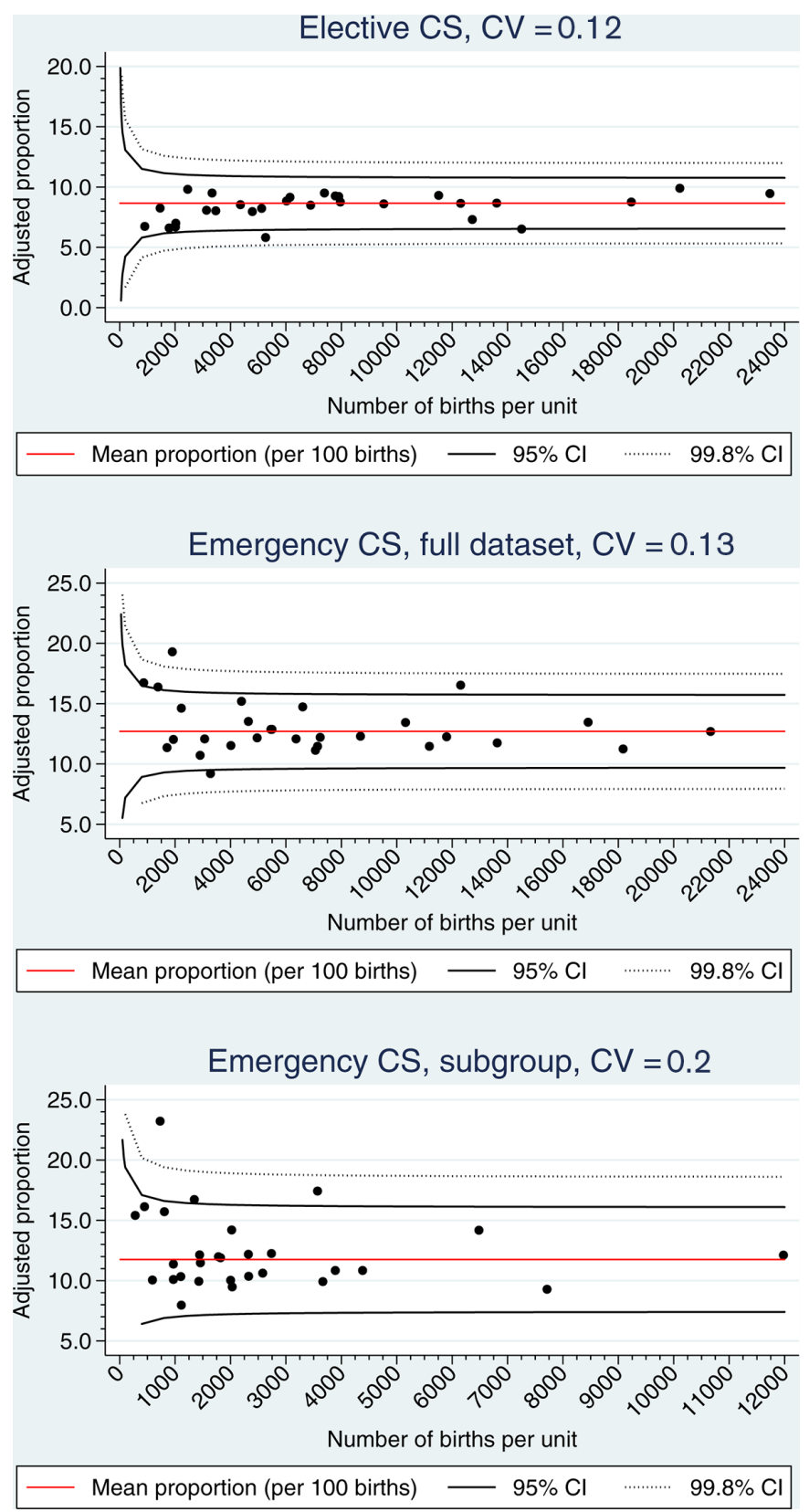

Figure 1 Elective CS (shown in the upper part), emergency CS (the middle part), emergency CS in a low-risk subgroup (the lower part). Risk-adjusted proportions of 29 maternity units, 2009-2012. Funnel plot limits: 95\% and 99.8\%. $\mathrm{CS}$, caesarean section; CV, coefficient of variation.

differed for night delivery (a significantly higher risk for emergency CS at night in the subgroup; OR 1.05 (95\% CI 1.0 to 1.1 ) versus 0.93 (95\% CI 0.9 to 0.95 ) in the full dataset), augmentation of labour (1.57 (95\% CI 1.49 to 1.66) versus 1.01 (95\% CI 0.98 to 1.05$)$ ) and macrosomia (3.36 (95\% CI 2.92 to 3.87 ) versus 2.58 (95\% CI 2.38 to 2.79)) .

\section{Between-hospital variation of risk-adjusted CS proportions}

The median of the observed proportion of elective CS in maternity units was $8.5 \%$ with a minimum of $3.6 \%$ and a maximum of $10.6 \%$. Risk-adjusted proportions ranged from $5.8 \%$ to $9.9 \%$ (median $8.6 \%, 25 \%-75 \%$ percentiles: 8.0-9.2) (figure 1, upper part). There was statistically significant heterogeneity among maternity units $(\mathrm{P}<0.001)$. Allowing for little overdispersion $(1.1 \%)$, no hospital maternity unit was outside the upper $95 \%$ funnel limit. However, one medium-sized unit was below the corresponding lower limit, indicating that this unit performed systematically fewer elective CS than expected.

The median of the observed proportion of emergency CS in maternity units was $12.7 \%$ with a minimum of $7.0 \%$ and a maximum of $17.1 \%$. Risk-adjusted proportions ranged from $9.2 \%$ to $19.3 \%$ (median $12.2 \%, 25 \%-75 \%$ percentiles: 11.5-13.5) (figure 1, middle part). There was statistically significant heterogeneity among the 29 maternity units $(\mathrm{P}<0.001)$. Taking the estimated overdispersion $(1.5 \%)$ into account, there were few maternity units outside the $95 \%$ funnel limits: one small unit was below the $95 \%$ lower limit and four units (two small, one medium and one large) were above the $95 \%$ upper limit, indicating that the latter units systematically performed more emergency CSs than expected.

In the emergency subgroup, the observed emergency CS proportions ranged from $7.8 \%$ to $19 \%$ (median $11.6 \%$ ) between maternity units, and risk-adjusted emergency CS proportions ranged from $8 \%$ to $23.2 \%$ (median $11.5 \%, 25 \%-75 \%$ percentiles: $10.1-14.2$ ) (figure 1, lower part). Risk-adjusted proportions showed statistically significant heterogeneity among maternity units $(\mathrm{P}<0.001)$. No hospital maternity unit was below the lower $95 \%$ funnel limit (overdispersion 2.2\%). However, two medium-sized units and one large unit were above the corresponding upper limit, indicating systematically more emergency CSs than expected.

\section{DISCUSSION}

Our study showed that mostly all of the included risk factors had an influence on the risk of CS. Breech presentation and previous CS were found to have the largest impact on elective CS; on emergency CS, it was breech presentation and cephalopelvic disproportion. As the proportions of elective and emergency CS were stable throughout the 4-year study period, the steep increase of the overall CS proportion in Denmark seems to have stopped. As for organisations, large hospital units performed significantly more elective CSs than smaller units, and the risk and performance of emergency CS was significantly reduced in the larger maternity units compared with small and medium units, which would be expected since there is a centralisation of risk births (eg, congenital anomalies, significant maternal comorbidity) in Denmark. For referral to delivery in smaller hospitals in Denmark, there are specific criteria such as normal pregnancy at term, uncomplicated pregnancy and delivery at term, whereas more complicated cases (eg, sick fetus, maternal comorbidities, preterm delivery and high maternal BMI) are referred to larger hospitals, leading to a higher rate of CS. We also found substantial 
heterogeneity of risk-adjusted CS between Danish maternity units, with higher variation in emergency CS than in elective CS. Even though we applied exhaustive regression models covering a wide range of risk factors, the results still indicated the presence of systematic, that is larger than random, variation between hospital units.

Our study had all the limitations inherent to a historical registry-based cohort study design, where not all potential maternal or neonatal risk factors were available. Coding was done after the end of delivery, implying that the registration of diagnoses for which there are no precise definitions, for instance, cephalopelvic disproportion, ${ }^{17}$ might not have been adequate. This has to be taken into consideration when comparing results across studies. For example, based on ICD-10 codes, only $2 \%$ of the women in our study had a reported cephalopelvic disproportion, whereas a prior study from the UK showed that cephalopelvic disproportion was the primary indication for CS in $35 \%$ of the women. ${ }^{23} \mathrm{~A}$ further limitation is the small size of Denmark, with fewer than 60000 births per year and less than 30 maternity units, making it statistically more challenging to detect outliers. Data for this study come from one country in Northern Europe only, and Denmark is a small, well-regulated country with equal and free access to health services. Even though data homogeneity might be regarded as strength, generalisability of the results to other countries is limited.

It is possible that most of the risk factors in our study may not have had an independent, direct causal effect on the CS proportion, even if they were highly significant. For example, the risk factor of labour induction ${ }^{24}$ may be linked to a latent unknown status of 'problematic delivery', which in turn is linked to CS probability. The lack of knowledge about the decision for CS is another weakness of this study. Specifically, the term 'emergency CS' covers a broad range of situations in a maternity ward, as emergency CS is seen as a homogenous group, and distinctions are not made between degrees of urgency. ${ }^{25}$ Some CSs are performed immediately because of a life-threatening situation, and others are performed for other, non-life-threatening reasons. Former studies have shown that the CS proportions are influenced by a variety of different settings such as the use of fetal monitoring, partograms or the active management of labour. ${ }^{25-27}$ As a first step, the quality of registration in the Medical Birth Register will improve when the degree of urgency is included. ${ }^{28}$

To ensure the same quality of care for all women (in Denmark), there should not be any systematic differences in CS decision making between maternity units, and thus such systematic variation between units should be eliminated. Starting points for such a process could be (1) a retrospective inspection of the hospital birth records of the women in our study who were classified as low risk but still underwent CS, which could shed light on the premises for CS decisions, and (2) a comparative prospective study with a specific focus on the precise circumstances influencing the decision to perform a CS (when, why, who) and including units with both fewer and more CSs than expected, which would enable the identification of important key factors that were missed in our study. Besides acquiring more clinical details, the role of women's preferences and the various ways of making clinical decisions in different areas need to be investigated more thoroughly. The WHO recommends that other relevant outcomes such as short and long-term maternal and paediatric outcomes (including stillbirths and breastfeeding) as well as the psychological or social well-being of both mother and child after birth ${ }^{9}$ should be included in future studies. Many obstetricians regard the CS proportion of $20 \%$ as too high, ${ }^{23}$ and with clearer consensus on when to perform CS, there will be an enhanced possibility of reducing the CS proportion without compromising the neonatal morbidity or mortality. Recent years have seen the development of a national cardiotocography education programme in Denmark with the aim of reducing fetal distress. ${ }^{29}$ It has been implemented in all maternity units in the country and includes the education and certification of midwives and obstetricians in cardiotocography.

\section{CONCLUSION}

Our study showed that the risk of CS is influenced by several risk factors and also confirmed well-known risk factors. The largest impact on elective CS was found to be breech presentation and previous CS; on emergency CS, it was breech presentation and cephalopelvic disproportion. We also found a variation in risk-adjusted CS between Danish hospitals, which was unexpected for such a small, well-regulated country like Denmark. The prior increase of the overall CS proportion in Denmark seems to have stopped, as the proportions of elective and emergency CS were stable throughout the study period. To ensure the same quality of care across the country, CS practices in hospital maternity units should be compared and, if possible, harmonised. Furthermore, the possible lack of consensus about clinical practice as well as the attitudes of parents and healthcare professionals towards mode of delivery should be investigated further.

Acknowledgements The main author wishes to thank Annett Helleskov Rasmussen for vital support regarding the study.

Contributors SW, RG and BMN devised the concept of the study. SW did the data management and data analysis. RG, SW and BMN drafted the article. All authors revised the manuscript and approved the final version.

Funding This research received no specific grant from any funding agency in the public, commercial or not-for-profit sectors.

Competing interests None declared.

Patient consent Not required.

Ethics approval The study was approved by the Danish Data Protection Agency (no. 2013-41-1561).

Provenance and peer review Not commissioned; externally peer reviewed.

Data sharing statement No additional data available.

Open Access This is an Open Access article distributed in accordance with the Creative Commons Attribution Non Commercial (CC BY-NC 4.0) license, which permits others to distribute, remix, adapt, build upon this work non-commercially, 
and license their derivative works on different terms, provided the original work is properly cited and the use is non-commercial. See: http://creativecommons.org/ licenses/by-nc/4.0/

(C) Article author(s) (or their employer(s) unless otherwise stated in the text of the article) 2018. All rights reserved. No commercial use is permitted unless otherwise expressly granted.

\section{REFERENCES}

1. Grivell RM, Dodd JM. Short- and long-term outcomes after cesarean section. Expert Rev Obstet Gynecol 2011;6:205-15.

2. Colmorn LB, Krebs L, Klungsøyr K, et al. Mode of first delivery and severe maternal complications in the subsequent pregnancy. Acta Obstet Gynecol Scand 2017;96:1053-62.

3. Villar J, Carroli G, Zavaleta N, et al. Maternal and neonatal individual risks and benefits associated with caesarean delivery: multicentre prospective study. BMJ 2007;335:1025.

4. Shorten A. Maternal and neonatal effects of caesarean section. BMJ 2007;335:1003-4.

5. Humberg A, Härtel C, Paul P, et al. Delivery mode and intraventricula hemorrhage risk in very-low-birth-weight infants: observational data of the German Neonatal Network. Eur J Obstet Gynecol Reprod Biol 2017;212:144-9.

6. Bragg F, Cromwell DA, Edozien LC, et al. Variation in rates of caesarean section among English NHS trusts after accounting for maternal and clinical risk: cross sectional study. BMJ 2010;341:c5065.

7. Clark SL, Belfort MA, Hankins GD, et al. Variation in the rates of operative delivery in the United States. Am J Obstet Gynecol 2007:196:526.e1-5.

8. Sydsjö G, Sydsjö A, Brynhildsen J, et al. Trends in caesarean section and instrumental deliveries in relation to Body Mass Index: a clinical survey during 1978 - 2001. Reprod Health 2010;7:18.

9. World Health Organization. WHO statement on caesarean section rates. Geneva: World Health Organization, 2015. (WHO/ RHR/15.02).

10. Althabe F, Belizán JM. Caesarean section: the paradox. Lancet 2006;368:1472-3.

11. Betrán AP, Merialdi M, Lauer JA, et al. Rates of caesarean section: analysis of global, regional and national estimates. Paediatr Perinat Epidemiol 2007;21:98-113.

12. Fødsler. Web-portal for interactive data exploration and retrieval on Births: 1997. 1997 http://www.esundhed.dk/sundhedsregistre/MFR/ Sider/MFR06A.aspx (accessed 26 Feb 2016).

13. Fødsler. Web-portal for interactive data exploration and retrieval on Births: 1973. 1973 http://www.esundhed.dk/sundhedsregistre/MFR/ Sider/MFR01.aspx (accessed 26 Feb 2016).

14. Poobalan AS, Aucott LS, Gurung T, et al. Obesity as an independent risk factor for elective and emergency caesarean delivery in nulliparous women--systematic review and meta-analysis of cohort studies. Obes Rev 2009;10:28-35.

15. Macfarlane AJ, Blondel B, Mohangoo AD, et al. Wide differences in mode of delivery within Europe: risk-stratified analyses of aggregated routine data from the Euro-Peristat study. Br J Obstet Gynaecol 2016;123:559-68.

16. García-Armesto S, Angulo-Pueyo E, Martínez-Lizaga N, et al. Potential of geographical variation analysis for realigning providers to value-based care. ECHO case study on lower-value indications of C-section in five European countries. Eur J Public Health 2015;25(Suppl 1):44-51.

17. Librero J, Peiró S, Calderón SM. Inter-hospital variations in caesarean sections. A risk adjusted comparison in the Valencia public hospitals. J Epidemiol Community Health 2000;54:631-6.

18. Nippita TA, Lee YY, Patterson JA, et al. Variation in hospital caesarean section rates and obstetric outcomes among nulliparae at term: a population-based cohort study. BJOG 2015;122:702-11.

19. Kozhimannil KB, Arcaya MC, Subramanian SV. Maternal clinical diagnoses and hospital variation in the risk of cesarean delivery: analyses of a National US Hospital Discharge Database. PLoS Med 2014;11:e1001745

20. Robson MS. Classification of caesarean sections. Fetal Matern Med Rev 2001;12:23-39.

21. Spiegelhalter DJ. Funnel plots for comparing institutional performance. Stat Med 2005;24:1185-202.

22. DerSimonian R, Laird N. Meta-analysis in clinical trials. Control Clin Trials 1986;7:177-88

23. Thomas J, Paranjothy S. Royal College of Obstetricians and Gynaecologists Clinical Effectiveness Support Unit: the National Sentinel Caesarean Section Audit Report. London: RCOG Press, 2001.

24. Yogev $Y$, Hiersch L, Yariv O, et al. Association and risk factors between induction of labor and cesarean section. J Matern Fetal Neonatal Med 2013;26:1733-6.

25. National Collaborating Centre for Women's and Children's Health, The National Institute for Health and Clinical Excellence. Caesarean section. NICE Clinical Guideline. London: RCOG Press, 2011.

26. Alfirevic Z, Devane D, Gyte GML. Continuous cardiotocography (CTG) as a form of electronic fetal monitoring (EFM) for fetal assessment during labour. Cochrane Database of Systematic Reviews 2006;3:CD006066.

27. Brown HC, Paranjothy S, Dowswell $\mathrm{T}$, et al. Package of care for active management in labour for reducing caesarean section rates in low-risk women. Cochrane Database of Systematic Reviews 2008:4:CD004907.

28. Kesmodel US, Jølving LR. Measuring and improving quality in obstetrics--the implementation of national indicators in Denmark. Acta Obstet Gynecol Scand 2011;90:295-304.

29. Thellesen L, Hedegaard M, Bergholt T, et al. Curriculum development for a national cardiotocography education program: a Delphi survey to obtain consensus on learning objectives. Acta Obstet Gynecol Scand 2015;94:869-77. 